\title{
A glycoside analog of mammalian oligomannose formulated with a TLR4-stimulating adjuvant elicits HIV-1 cross-reactive antibodies
}

\section{Jean-Francois Bruxelle ( $\nabla$ jbruxell@sfu.ca )}

Faculty of Health Sciences, Simon Fraser University, Burnaby, BC, Canada

\section{Tess Kirilenko}

Faculty of Health Sciences, Simon Fraser University, Burnaby, BC, Canada

\section{Nino Trattnig}

Department of Chemistry, University of Natural Resources and Life Sciences, Vienna, Austria

\section{Yiqiu Yang}

Department of Molecular Biology \& Biochemistry, Simon Fraser University, Burnaby, BC, Canada

\section{Matteo Cattin}

Department of Chemistry, University of Natural Resources and Life Sciences, Vienna, Austria

\section{Paul Kosma}

Department of Chemistry, University of Natural Resources and Life Sciences, Vienna, Austria

\section{Ralph Pantophlet ( $\square$ rpantophlet@sfu.ca )}

Faculty of Health Sciences, Department of Molecular Biology \& Biochemistry, Simon Fraser University, Burnaby, BC, Canada

\section{Research Article}

Keywords: Antibody, Oligomannose, Glycoconjugate, Adjuvant, HIV

Posted Date: January 6th, 2021

DOI: https://doi.org/10.21203/rs.3.rs-140631/v2

License: (1) (i) This work is licensed under a Creative Commons Attribution 4.0 International License. Read Full License 


\section{Abstract}

The occurrence of oligomannose-specific broadly neutralizing antibodies (bnAbs) has spurred efforts to develop immunogens that can elicit similar antibodies. Here, we report on the antigenicity and immunogenicity of a $\mathrm{CRM}_{197}$-conjugate of a previously reported oligomannose mimetic. Oligomannosespecific bnAbs that are less dependent on interactions with the HIV envelope protein sequence showed strong binding to the glycoconjugates, with affinities approximating those reported for their cognate epitope. The glycoconjugate is also recognized by germline precursors of oligomannose-specific bnAbs, albeit with the expected low avidity, supporting its potential as an immunogen. Immunization of humanantibody transgenic mice revealed that only a TLR4-stimulating adjuvant formulation evoked antibodies able to bind a panel of recombinant HIV trimers. These antibodies bound at relatively modest levels, likely explaining their inability to neutralize HIV infectivity. Nevertheless, these findings contribute further to understanding conditions for eliciting HIV-cross-reactive oligomannose-specific antibodies and inform on next steps for improving on the elicited response.

\section{Introduction}

An effective vaccine is still considered the best way to blunt the global spread of HIV/AIDS. However, the design of such a vaccine continues to face scientific challenges, including the development of a component that can elicit broadly reactive HIV-neutralizing antibodies (bnAbs). Extensive investigations have led to the delineation of seven antigenic sites on the HIV-1 envelope spike that are targets of bnAbs, among which is a relatively conserved patch of oligomannose-type glycans ${ }^{1}$. NAbs to this glycan patch are among the most potent and cross-reactive ${ }^{2}-$ a recognition that has incentivized attempts to design immunogens capable of eliciting equivalent antibodies.

A common belief is that the elicitation of antibodies to the aforementioned glycan patch is likely to be hampered by B cell tolerance mechanisms, because of the 'self' nature of targeted molecules. One potential way to overcome tolerogenic restrictions is to engineer glycosides that appear 'foreign' to the immune system but that sufficiently mimic oligomannose to yield cross-reactive antibodies. Not long ago, we began probing this idea based on the discovery of a bacterial oligosaccharide with resemblance to oligomannose ${ }^{3,4}$ and knowledge that mimicry of mammalian oligosaccharides by bacterial glycans can overcome natural tolerance. We previously reported on a neoglycoside that when conjugated to BSA elicits modest levels of nAbs against select HIV strains following immunization of human-antibody transgenic rats ${ }^{5}$ and are working on strategies to heighten elicitation of the desired antibodies, for example by introducing distinctive bacterial constituents ${ }^{6}$.

One of the caveats of our previous study was the use of BSA as a carrier protein. Although convenient, BSA is not a clinically acceptable carrier. To address this caveat, we have chosen $\mathrm{CRM}_{197}$, a non-toxic mutant of diphtheria toxin. $\mathrm{CRM}_{197}$ is now common in marketed glycoconjugate vaccines and 
is known to stimulate robust T-follicular helper cell (Tfh) responses ${ }^{7-10}$, a feature that is important for affinity maturation of anti-glycan Abs ${ }^{11}$. We report here on the binding interaction of oligomannosespecific bnAbs from several different families along with their inferred germline precursors to a CRM 197 conjugate of our lead glycoside ${ }^{5}$.

Another limitation of our previous study was the choice of adjuvant. Several adjuvants are now used in human vaccines ${ }^{12}$, albeit that only alum and MF59 are used in current glycoconjugate vaccines. MF59 is better than alum with some glycoconjugate vaccines ${ }^{13}$ and therefore an MF59-like formulation (Addavax; Invivogen) was used in our previous work. However, because MF59 does not work well with all glycoconjugates ${ }^{14}$, we wished to evaluate how different categories of clinically relevant adjuvant formulations might impact the immunogenicity of our glycoside conjugate. Specifically, we report here on the efficacy of adjuvants exemplifying three different categories-aluminum salt (Alhydroge ${ }^{\circledR}$ ), squalene oil-in-water (AddaVax ${ }^{T M}$ ), TLR4 agonist (GLA-SE) ${ }^{15-17}$ - when formulated with our glycoconjugate to induce HIV-cross-reactive antibody responses. Here, we used the Trianni mouse model, which express a full human antibody repertoire, to evaluate the immunogenicity of our conjugate in animals that evolve human-like antibody responses ${ }^{18}$. We show that only the immunization with the oligomannose mimetic conjugate formulated in GLA-SE elicited antibody responses with capacity to bind the oligomannose mimetic. Sera from these animals also bound various recombinant HIV SOSIP gp140s. However, analysis of this antibody response revealed that these two responses are distinct, with those specific for the mimetic being predominantly of the IgG3 subclass whereas those specific for HIV Env were largely IgG2. Although immunizing repeatedly with the same conjugate was not sufficient to yield detectable nAbs, our findings provide clues for possible ways to augment the level of cross-reactivity to achieve virus neutralization.

\section{Results}

\section{Members of the oligomannose-patch specific PGT128/130 bnAb family bind avidly to $\mathrm{CRM}_{197^{-}}$ conjugated glycomimetic NIT211, with affinities approximating those reported for recombinant HIV.}

We first evaluated by ELISA the binding of oligomannose-specific bnAbs from the PGT128/130 family (PGT125, 126, 128, 130) to the $\mathrm{CRM}_{197}$-conjugated glycoside, dubbed NIT211. We had used these bnAbs previously for antigenic characterization of the BSA conjugate of the same glycoside ${ }^{5}$. All four antibodies bound NIT211 (4.1 glycans per $\mathrm{CRM}_{197}$ ) avidly ( $\mathrm{EC}_{50} 0.1$ - 7 nM; Fig. 1A). As reported recently ${ }^{19}$, these antibodies bind NIT211 at least as good as the BSA conjugate loaded at similar density.

We then used recombinantly expressed Fab fragments in conjunction with Biacore SPR to determine binding affinities of the four PGT antibodies for NIT211. A biotinylated version of NIT211 (4.1 glycans per $\mathrm{CRM}_{197}$ ), captured onto the sensor surface by immobilized streptavidin, served as the ligand. Results show that the Fabs bind NIT211 with average affinities ranging from $271 \mathrm{nM}$ (PGT130) to $678 \mathrm{nM}$ (PGT128) (Table 1, Fig. 2A). The $\mathrm{K}_{\mathrm{D}}$ values determined here for PGT125 and PGT128 are similar to the 
reported monovalent $K_{D}$ values of these antibodies for a Man $_{9}-V 3$ glycopeptide (PGT125: 706 nM; PGT128: $326 \mathrm{nM}$ ) and the estimated monovalent $K_{D}$ of PGT128 for BG505 SOSIP (303 nM) ${ }^{20}$. Similar to others ${ }^{21}$, we observed biphasic sensorgrams for all the PGT antibodies despite the use of monovalent Fab molecules, suggestive of a bivalent binding interaction with NIT211 (Fig. 2B).

Oligomannose-specific bnAbs such as those from the PGT128/130 family interact with two or more glycans on HIV Env ${ }^{22,23}$. To evaluate the relationship between NIT211 glycan density and antibody binding avidity, and given the above-noted observations of biphasic antibody binding in SPR experiments, we assayed by ELISA binding of the PGT antibodies to NIT211 conjugated with the oligomannose mimetic at densities of 2.6, 4.1, and 6.2 glycans per $\mathrm{CRM}_{197}$. We first assessed binding of the antibodies formatted as IgGs. Consistent with our previous report ${ }^{5}$, antibodies of the PGT128/130 family bound increasingly avid to NIT211 with increasing glycan density (Fig. 3A), with apparent maxima at a density of $\sim 6$ glycosides. Strikingly, the relative binding affinity of the Fab fragments of antibodies PGT125, 126 and 128 also increased substantially upon glycan density (Fig. 3B) suggesting a bivalent or greater interaction of these Fab fragments to NIT211 ligands. Taken together with the SPR analyses, these results suggest that presentation of the oligomannose mimetic at a density of 4-6 molecules per $\mathrm{CRM}_{197}$ reasonably approximates oligomannose presentation on HIV gp120 conducive to the binding of at least the PGT128/130 family of bnAbs.

\section{Oligomannose patch-specific bnAbs outside of the PGT128/130 bnAb family bind less avidly to $\mathrm{CRM}_{197^{-}}$ glycoconjugate NIT211.}

Having established that our lead glycomimetic conjugated to $\mathrm{CRM}_{197}$ retains favorable antigenicity for members of the PGT128/130 bnAb family as compared to the BSA conjugate, we next evaluated binding of representatives from other bnAb families specific for the oligomannose patch (BF520.1, BG18, PCDN33A, PGDM12, PGDM21, VRC41.01) 20,24-27. We found that bnAbs BG18, PGDM21, and VRC41.01 bind NIT211 (4.1 glycans per $\mathrm{CRM}_{197}$ ) with reasonably high avidity ( $\mathrm{EC}_{50} 14-70 \mathrm{nM}$ ) (Fig. 1B), albeit less than the PGT128/130 bnAbs. In contrast, bnAbs BF520.1, PCDN-33A and PGDM12 bound the CRM 197 conjugate very poorly ( $\left.E_{50} 1-5 \mu \mathrm{M}\right)$ (Fig. 1B). Binding of these antibodies as Fab fragments was not detectable by SPR. Overall, these results likely reflect the varied degrees of specificity of the bnAbs for oligomannose, their capacity to engage other glycans, and their relative dependence on interaction with the $\mathrm{V} 3$ protein backbone. Nevertheless, the results suggest that the oligomannose mimetic and its presentation on $\mathrm{CRM}_{197}$ sufficiently imitates the binding epitope of not just the PGT128/130 bnAb family but also a few other oligomannose-specific bnAbs.

$\mathrm{CRM}_{197^{-}}$-conjugated glycoside mimic of oligomannose is bound also by inferred germline (gl) precursors of anti-HIV oligomannose-specific bnAb families. 
A prevailing thesis in the HIV vaccine field currently is that an immunogen that can be bound with sufficient affinity in vitro by gl precursors of existing bnAbs might be better able to activate the appropriate B cells in vivo to yield similar bnAbs (reviewed in ref. ${ }^{1}$ ). We therefore assessed the binding of published inferred gl precursors from four oligomannose-specific bnAb families (BF520.1, BG18, PCDN33A and PGT128/130) for binding in ELISA to NIT211 (4.1 glycans per CRM 197 ). As shown in Fig. 1C, all four $\mathrm{gl}$ antibodies bound NIT211, albeit with the expected lower avidity $\left(\mathrm{EC}_{50} 0.1-5 \mu \mathrm{M}\right)$ compared to their mature cognates. Fab fragments of these gl antibodies did no bind detectably to NIT211 by SPR. Recognition of the conjugated oligomannose mimetic by various oligomannose bnAbs and, most importantly, some of their respective gl precursors provided encouragement in NIT211's potential to trigger B cells with nominal specificity for oligomannose.

\section{$\mathrm{CRM}_{197}$-conjugated glycoside NIT211 elicits glycan-specific antibodies in human-antibody transgenic Trianni mice.}

Having determined that the NIT211 glycoconjugate is bound reasonably well by several oligomannosespecific bnAbs and a few gl versions thereof, we next sought to identify a possibly optimal adjuvant formulation for the elicitation of the desired glycan-specific antibody response. We chose to conduct our immunizations in the human-antibody transgenic Trianni mouse system to allow for an approximation of possible antibody responses in people. Before commencing, we confirmed that these mice do not develop an abnormal antibody response by subcutaneously administering a priming immunization with the model antigen KLH formulated in Alhydrogel plus CpG ODN1826. We found total IgG responses in Trianni mice to be as robust as similarly primed wild-type C57BL/ 6 mice at one month post-immunization (Supplementary Fig. 1A), albeit that the $\lg \mathrm{G} 1$ and IgG2c responses in Trianni were notably stronger (Supplementary Fig. 1B). Total T-follicular helper (Tfh) cell frequencies (B220-CD $4^{+} \mathrm{CD} 44^{\mathrm{hi}} \mathrm{PD} 1^{+} \mathrm{CXCR} 5^{+}$) in draining inguinal lymph nodes at day 8 post prime (4.9-13.1\%) were also similar to the average frequencies reported in the draining popliteal lymph nodes of non-transgenic mice ${ }^{28}$ after a single footpad immunization with KLH formulated with alum (Supplementary Fig. 2).

We then assessed and compared the immunogenicity of NIT211 formulated in three different types of adjuvants: Alhydrogel, AddaVax ${ }^{\mathrm{TM}}$, and GLA-SE. The NIT211 conjugate used for immunization carried an average of 6.2 glycosides per $\mathrm{CRM}_{197}$ molecule, which, as shown in Fig. 3A, was bound strongly by the PGT antibodies. Mice ( $n=5$ per group) were primed at day 0 and boosted at days 21,42 and 105 , a schedule modeled on glycoconjugate vaccine schedules in humans ${ }^{29,30}$, and sera collected on days 0,10 , 28,49 , and 119.

All mice immunized with the GLA-SE formulation mounted a rapid IgG response to the $\mathrm{CRM}_{197}$ carrier protein by day 10 after priming (Supplementary Fig. 3), consistent with previous reports ${ }^{19}$ and the expected high immunogenicity of the carrier. The magnitude of the antibody response was somewhat slower in mice immunized with the Alhydrogel and AddaVax formulations. Even so, IgG levels in all three 
animal groups plateaued following the second boost (day 49; Supplementary Fig. 3). Overall, mice immunized with the GLA-SE formulation produced the highest IgG response to the $\mathrm{CRM}_{197}$ carrier protein, followed by the AddaVax and Alhydrogel groups, respectively.

To measure antibody responses to the oligomannose mimetic without interference from antibodies specific for the $\mathrm{CRM}_{197}$ carrier, we used the BSA-conjugated version of the glycoside (dubbed NIT82b5), which was conjugated at 4 glycosides per BSA molecule. We confirmed that none of the sera from the final bleed (day 119) bound unconjugated BSA (Supplementary Fig. 4), meaning that any measured binding to NIT82b should be due to the conjugated glycoside. Unexpectedly, we found that only mice immunized with the GLA-SE adjuvanted conjugate produced IgG that bound the oligomannose mimetic as presented on BSA (Fig. 4A). We found no evidence of glycoside-specific IgM antibodies in sera from any of the three groups of immunized animals (Supplementary Fig. 5). The level of IgG binding varied among the GLA-SE-immunized mice; two of the animals produced reasonably good anti-glycan antibodies, a third produced modest levels and two appeared to not produce much antibody (Fig. 4B). Strikingly, IgG3 was the most prevalent IgG subclass in the response of GLA-SE immunized animals to the oligomannose mimetic, whereas $\lg G 1$ and $\lg G 2$ (IgG2b and IgG2c) were the most prevalent against the $\mathrm{CRM}_{197}$ carrier (Fig. 4C).

In sum, the results above show that NIT211 formulated in GLA-SE induced the greatest antibody response to the glycomimetic compared to formulations with Alhydrogel or Addavax.

\section{Anti-glycan serum antibodies elicited by $\mathrm{CRM}_{197}-$ glycoconjugate NIT211 in GLA-SE bind recombinant HIV gp140 trimers but are unable to exert neutralizing activity.}

We next assessed whether the elicited anti-glycan antibody response could recognize glycans on HIV. First, we measured serum IgG binding to a panel of SOSIP-based HIV gp140 trimers by ELISA. Unexpectedly, notable lgG binding was observed with the sera of all five animals in the GLA-SE group (Fig. 5A), including sera from the two animals that had bound only marginally to the BSA conjugate. This unexpected result prompted us to also assay sera from the animals immunized with the Alhydrogel and Addavax formulations. Sera from these animals did bind somewhat to a selection of SOSIP trimers, but this binding was similar to the binding of sera from unimmunized mice to the trimers, suggesting that it may have been caused by naturally occurring anti-glycan antibodies. We observed binding of all the GLASE sera to the B41 SOSIP.v4.1 trimer (Fig. 5A), which was chosen for this analysis.

IgG subclass analyses revealed that whereas IgG3 was the predominant IgG subclass responsible for binding to the oligomannose mimetic in sera from animals immunized with the GLA-SE formulation (cf. Fig. 4C), serum antibodies binding to the SOSIP trimers were predominantly lgG2 (Fig. 5B). These results suggest that the immunization with NIT211 yielded two different anti-glycan responses; one, mainly of 
the IgG3 subclass, specific only for the synthetic glycoside, and the other, mainly of the lgG2b/c subclass, capable of binding glycans on HIV.

To determine whether the NIT211 + GLA-SE formulation had truly stimulated antibodies with capacity to bind the high-mannose patch on HIV gp120, we performed an inhibition ELISA assay in which we measured the binding of bnAb PGT128 to B41 SOSIP trimer after incubation with GLA-SE sera vs immune sera from animals immunized with $\mathrm{KLH}$, which itself carries a large number of high mannose-type glycans ${ }^{31}$. We observed a notable decrease in PGT128 residual binding following incubation with the NIT211 + GLA-SE sera compared to KLH immune sera (Fig. 5C). These results show that NIT211, when formulated with GLA-SE, had indeed elicited antibodies capable of binding glycans within or neighboring the high-mannose patch.

Given the observed binding of the NIT211 + GLA-SE sera to SOSIP trimers, we assessed the sera for neutralizing activity against a small panel of HIV-1 strains from different subtypes in the Monogram PhenoSense neutralization assay. However, we observed no significant neutralizing activity relative to unimmunized animals even at the lowest serum dilution tested (1:30) (Fig. 5D). We conclude from these results that the elicited antibodies are of insufficient affinity (or avidity) to bind glycans on the virus or at least not enough to exert neutralizing activity.

\section{Discussion}

In this study we have identified the TLR4 agonist adjuvant GLA-SE as conducive to the elicitation of glycan-specific HIV-binding antibodies by NIT211, a $\mathrm{CRM}_{197}$ conjugated oligomannose mimetic. Of note is that we were able to elicit the aforementioned antibodies in animals expressing a full human antibody repertoire, demonstrating the ability of the presented mimetic to be sensed by naïve $B$ cells of the desired specificity in a milieu that is not necessarily favorable. Although the elicited antibodies did not bind the virus with sufficient affinity or avidity to exert neutralizing activity, we consider the ability of the elicited antibodies to bind recombinant trimers encouraging.

Our analyses of the antigenic characteristics of NIT211 led to several notable observations. First, the apparent binding affinity of PGT128 and several related antibodies for our neoglycoconjugate increased with increasing glycan density (Fig. 3), which suggests that increased glycan density results in the presentation of oligomannosides in proximity to a peptide backbone that may resemble the PGT epitope better and therefore enhance antibody binding. The close binding affinity of Fab PGT128 for NIT211 (0.6 $\mu \mathrm{M}$; Fig. 2) and its estimated affinity for SOSIP gp140 $\left(0.3 \mu \mathrm{M}^{20}\right)$ suggests that presentation of the mimetic on $\mathrm{CRM}_{197}$ at least somewhat imitates the organization of oligomannose residues on Env to which PGT128 and related antibodies bind.

In addition to strong binding by members of the PGT128/130 family, we also observed reasonably avid binding by the oligomannose-specific bnAbs VRC41.01, PGDM21 and BG18 (Fig. 1B), suggesting that presentation of the mimetic on $\mathrm{CRM}_{197}$ also somewhat resembles their respective cognate epitopes on 
HIV Env. However, other antibodies (BF520.01, PGDM12 and PCDN-33A) bound poorly. Different factors may be limiting the ability of these antibodies to bind more avidly. For example, the glycan binding profile of VRC41.01 ${ }^{20}$ suggests that this antibody binds an epitope involving the reducing end of $N$-linked oligomannose. The lack of a chitobiose core at the reducing end of our mimetic may be one reason for VRC41.01's inability to bind the mimetic more avidly. Another example is BG18, which binds a complex set of 3-4 oligomannose-type glycans on Env ${ }^{32}$ that is unlikely to be fully replicated on NIT211, thus perhaps explaining that antibody's inability to bind more avidly to the glycoconjugate. In contrast, PCDN antibodies such as PCDN-33A have been shown to bind only to $\mathrm{Man}_{9} \mathrm{GICNAc}_{2}{ }^{26}$, indicating strong dependence on the D2 arm of oligomannose - which is not replicated in the mimetic and therefore likely explains the poor binding of that antibody.

NIT211's potential for triggering the elicitation of oligomannose-specific antibodies is evident also from the binding of inferred gl precursors from several of the oligomannose-specific bnAbs that were assayed (BF520.1, BG18, PCDN-33A, PGT128/130) (Fig. 1C). We did note that binding of the PGT128/130 gl antibody was the least of all the antibodies assayed $\left(E_{50} 1.3 \mu \mathrm{M}\right)$. Given the strong binding of affinity matured descendants such as PGT128, these results underscore the extent to which this family of antibodies has evolved to gain affinity for oligomannose while maintaining promiscuity for different oligomannose structures ${ }^{33}$. In notable contrast, binding of the PCDN-33A gl antibody was the strongest of the four $\mathrm{gl}$ antibodies assayed $\left(\mathrm{EC}_{50} 0.2 \mu \mathrm{M}\right)$, yet binding of the affinity matured descendant was poor (Fig. 1B). PCDN-33A's binding specificity for full-sized oligomannose $\left(\mathrm{Man}_{9}\right)^{26}$ suggests that as the gl antibody matured, it lost its relative promiscuity by mutating away from being able to recognize lowerorder oligomannose structures.

Analyses of NIT211's immunogenicity also led to in a few notable and unexpected observations. First, although we found that the GLA-SE formulation yielded a balanced lgG1/lgG2 antibody response to the $\mathrm{CRM}_{197}$ carrier protein as expected ${ }^{16}$, we were struck by the IgG3 subclass response to the glycoside component (Fig. 4). This distinctive response is noteworthy given that IgG3 is a common subclass among bacterial oligosaccharide-specific antibody responses in mice ${ }^{34-36}$ and given that we designed our mimetic to appear foreign to the immune system ${ }^{5}$. We therefore interpreted these results as suggesting that the Trianni mouse immune system sensed our glycoconjugate as a bacterial polysaccharide, possibly aided by its formulation in a TLR4 agonist adjuvant. Unexpectedly however, these IgG3 responses did not cross-react with HIV-1 SOSIP trimers. Instead, our analyses revealed that a second glycan-specific antibody response, apparently of the lgG2 subclass, was induced also that is able to bind SOSIP trimers and, specifically, to glycans within or neighboring the high mannose patch (Fig. 5). Whether these subclass-distinctive responses developed independently parallel to each other or whether one developed after the other requires further investigation, as this may reveal an immunization time point at which to possibly boost the HIV-cross reactive antibody response. Nevertheless, as discussed further below, possible trimming of our glycoside mimetic by a soluble mannosidase during the first 
immunizations gives reason to believe that HIV-cross reactive antibodies likely developed later in the immunization process.

There is general belief that at least one major difficulty in eliciting oligomannose-specific bnAbs stems from B cell tolerance (reviewed in ${ }^{37}$ ). Synthetic oligomannose-like glycosides, such as the ones that we are pursuing $5,6,38$, are one potential avenue to overcome tolerance, based on the notion that anergic $B$ cells can be 'roused' by exposing them to seemingly cross-reactive antigens along with the proper immunostimulatory signals ${ }^{39}$. However, a thesis put forth recently by Nguyen et al. ${ }^{40}$ suggests that trimming of oligomannose-based conjugates upon immunization, perhaps rather than immune tolerance, may be impeding the elicitation of oligomannose-specific bnAbs. Our own investigations revealed that after prime and two boosters, sera of mice immunized with NIT211 + GLA-SE produced an IgG response that bound better to serum mannosidase-treated (i.e., trimmed) conjugate compared to the buffer-treated conjugate ${ }^{19}$. This might explain our inability to measure much of a response to the target glycoside until after the second booster (day 49; Fig. 4). We found however that the sera from NIT211 + GLA-SE immunized mice generally exhibited less preference for mannosidase-trimmed glycoside after the third boost (Supplementary Fig. 6A). These observations suggest that the immunization scheme used here allowed trimming of the administered glycoside by soluble mannosidase in vivo to be mitigated. Nevertheless, it will be of interest to determine whether a mimetic protected from mannosidase trimming yields greater HIV-reactive antibodies.

Another group ${ }^{41}$ has posited that nAb induction might be impaired due to the action of mannose-binding lectin (MBL). We did not determine MBL levels in the Trianni mice used here and therefore cannot fully exclude this possibility for our glycoconjugate. However, our ability to evoke glycan-specific antibodies, including those with the capacity to bind glycans on HIV, suggests that hindrance by MBL, if any, is not absolute. One possible explanation is that the MBL acute phase response in laboratory mice appears to peak already at $\sim 30 \mathrm{~h}^{42}$, which may allow enough immunogen to escape elimination by MBL.

In conclusion, we show here that our $\mathrm{CRM}_{197}$-conjugated oligomannose mimetic exhibits promising immunogenicity, as shown by the elicitation of antibodies that can bind the HIV high-mannose patch. Although these antibodies did not exert neutralization activity, our analyses help to inform on the kinetics of the desired response. An obvious next step is, for example, to evaluate whether boosting with a SOSIP trimer can generate higher-affinity antibodies.

\section{Experimental Methods}

\section{Glycoside synthesis and neoglycoconjugate preparation.}

The oligomannose mimetic was synthetized as previously described ${ }^{5}$. The BSA conjugate of the ligand, termed NIT82b, with an average loading density of 4 glycosides per BSA molecule, was also prepared as previously reported ${ }^{5}$. The $\mathrm{CRM}_{197}$ conjugates, collectively dubbed NIT211, at densities ranging from 2-6 glycosides per $\mathrm{CRM}_{197}$ molecule, were synthesized as described elsewhere recently ${ }^{19}$. Biotinylated 
NIT211 was generated with a sulfo-NHS biotinylation kit (ThermoFisher) in the same manner as reported previously for the BSA conjugate ${ }^{5}$.

\section{Recombinant antibody expression and purification}

PGT125, 126128 and 130 Fabs were expressed in FreeStyle ${ }^{\text {TM }}$ 293S cells (Life Technologies) grown in suspension by transfection at a 1:1 ratio of plasmids encoding light and heavy chain (truncated at $\mathrm{Asp}^{\mathrm{H} 234}$ ). Supernatants harvested 6 days after transfection were passed over an anti-human lambda affinity matrix (CaptureSelect ${ }^{\text {TM }}$ LC-lambda (hu); Thermo Scientific) equilibrated in PBS. Fab fragments were eluted with $0.1 \mathrm{M}$ glycine, pH 3.0 and neutralized with $10 \times$ concentrated PBS. Recombinant IgGs of all antibodies used in this study were expressed in FreeStyle ${ }^{\mathrm{TM}} 293 \mathrm{~F}$ cells and purified on a protein $\mathrm{A}$ resin (Thermo Scientific) using the aforementioned glycine solution for elution, as recently described ${ }^{19}$.

\section{Animal immunizations}

Trianni mice (6-8 weeks of age at the start of immunization) were immunized under contract at Antibody Solutions (Santa Clara, $C A$ ). Animals ( $n=5$ per group) were immunized subcutaneously (base of tail) with NIT211 conjugate formulated in $2 \%$ Alhydrogel $^{\circledR}$ (Invivogen), AddaVax ${ }^{\text {TM }}$ (Invivogen) or GLA-SE (IDRI). Anesthesia (isoflurane) was used to sedate animals during injections. Mice were primed at day 0 , and boosted at day 21, 42 and 105. Each mouse received $30 \mu \mathrm{g}$ of glycoconjugate, corresponding to $\sim 3 \mu \mathrm{g}$ of carbohydrate. Alhydrogel was mixed with antigen at a ratio of 2.5:1 (v/w), AddaVax ${ }^{\mathrm{TM}}$ with an equal volume of antigen, as per manufacturer's instructions, and GLA-SE was prepared by mixing each dose of antigen with $20 \mu \mathrm{l}$ each of an oil emulsion and a GLA emulsion, resulting in a dose of $5 \mu \mathrm{g}$ of GLA per animal per dose. All formulations were diluted in endotoxin-free TBS. The immunizations were approved by Simon Fraser University's Animal Care Committee (protocol. no. 1242HS-17). No notable adverse reactions were observed. A small bleed ( $<10 \%$ maximum blood volume) was collected via the saphenous vein from all animals just prior to immunization on day 0 , at day 10 post-prime, and then on days 28 and 49 after the first and second booster injections, respectively. The animals were exsanguinated following euthanasia on day 119, two weeks after the third booster injections, and splenectomized. Euthanasia was done by $\mathrm{CO}_{2}$ inhalation followed by cervical dislocation. All collected blood was left to clot and serum recovered, which was then frozen until required for analyses. Splenocytes were recovered from the collected spleens using Histopaque-1077. An additional group of 5 mice served as unimmunized controls and sampled in analogous manner.

\section{ELISA}


To assess binding of oligomannose-specific bnAbs and serum antibodies to the NIT211 glycoconjugate, the antigen was absorbed onto 96 -well polystyrene ELISA plates (Corning) at $5 \mu \mathrm{g} / \mathrm{ml}$ in PBS overnight at $4^{\circ} \mathrm{C}$. After washing, the plates were blocked with PBS supplemented with $3 \%(\mathrm{w} / \mathrm{v})$ casein (PBS-C). BnAbs and immune sera were serially diluted in PBS-C plus $0.02 \%(\mathrm{v} / \mathrm{v})$ Tween-20 (PBS-C-T). After washing, whole IgG bnAbs were detected with horseradish peroxidase (HRP)-conjugated anti-human Fc IgG (Jackson ImmunoResearch) and 3,3',5,5'-tetramethylbenzidine (TMB) (Ultra TMB, Thermo Scientific). Reactions were stopped after 5 minutes with $2 \mathrm{M}$ sulfuric acid and absorbances measured immediately at $450 \mathrm{~nm}$. Fabs were detected with CaptureSelect ${ }^{\mathrm{TM}}$ biotinylated anti-human $\mathrm{CH} 1$ antibody (Thermo Scientific; $0.2 \mu \mathrm{g} / \mathrm{ml}$ in PBS-C-T) in conjunction with alkaline phosphatase conjugated streptavidin (Jackson ImmunoResearch; $0.6 \mu \mathrm{g} / \mathrm{ml}$ in PBS-C-T) and nitrophenylphosphate substrate (Sigma). Reaction were developed for 30 minutes and absorbances measured immediately thereafter at $405 \mathrm{~nm}$. Total serum IgG was detected with a mixtures of equal amounts of alkaline phosphatase conjugated antimouse IgG1, IgG2b, IgG2C and IgG3 secondary antibodies (Jackson ImmunoResearch) and nitrophenylphosphate substrate (Sigma). For detecting individual subclasses, the aforementioned secondary antibodies were used alone. Reactions were allowed to develop for 30 minutes, after which absorbances were measured at $405 \mathrm{~nm}$. Serum mannosidase trimming experiments were performed as described elsewhere ${ }^{19}$.

To assess serum binding to SOSIP trimers, purified HIS-tagged trimers (kindly provided by John Moore (Weill Cornell Medical College) and Rogier Sanders (Amsterdam UMC)) were absorbed onto nickel-coated 96-well ELISA plates (Thermo Scientific) at $5 \mu \mathrm{g} / \mathrm{ml}$ in PBS overnight at $4^{\circ} \mathrm{C}$ and then blocked with PBS-C. The remaining steps for detection of serum antibody binding were performed as described above.

For inhibition ELISAs, B41 SOSIP.v4.1 HIS-tagged trimer was absorbed onto nickel-coated 96-well ELISA plates as described above. After blocking with PBS-C, sera were serially titrated in PBS-C-T onto the bound antigen. After washing, PGT128 was added at fixed concentration $(0.5 \mu \mathrm{g} / \mathrm{ml}$ in PBS-C-T $)$. After incubation (1 h) and washing, PGT128 residual binding was detected with HRP-conjugated secondary antibody and TMB substrate as described above.

\section{Antibody affinity measurements}

Antibody binding affinities were determined by surface plasmon resonance (Biacore X100) using Fab fragments and single cycle kinetics analysis. Biotinylated NIT211 (4.1 glycosides) was captured onto a sensor chip pre-immobilized with streptavidin using the biotin CAPture kit (400 RU). Fab fragment binding was determined usually serially titrated antibody, starting from $2.5 \mu \mathrm{M}$. All resonance signals were corrected by double referencing; signals monitored on the binding active channel were subtracted from the reference channel (sensor surface not modified with any reference protein) and by buffer signals. Data were globally fit to a 1:1 binding model within the BIA software. 


\section{Pseudovirus-based neutralization assays}

Sera were tested for virus neutralizing activity under contract at Monogram Biosciences (South San Francisco, CA) their pseudovirus-based neutralization assay with U87 target cells expressing CD4, CCR5, and CXCR4 as previously described ${ }^{43,44}$. A small panel of six HIV-1 strains (92TH021, 92RW020, 94UG103, 92BR020, 97ZA012, JRCSF) were assayed, chosen for their varied sensitivity to oligomannosespecific bnAbs ${ }^{45}$. Vesicular stomatitis virus $\mathrm{G}$ pseudotyped virus was used as a negative control because this glycoprotein does not express oligomannose on its surface ${ }^{46}$. The sera were assayed at a starting dilution of $1 / 30$. Serum neutralization titers were considered significant only if $\geq 3$ times greater than the values obtained for the negative control virus.

\section{Statistics}

Data were analysed using GraphPad Prism 9.0 software (San Diego, CA) and, unless otherwise stated, are presented as the mean with error bars denoting the standard error from the mean. Data for antibody binding experiments are from two or three experimental replicates. Serum antibody analyses represent variance among animals (5 per group) in a single experiment.

\section{References}

1. Kwong, P. D. \& Mascola, J. R. HIV-1 Vaccines Based on Antibody Identification, B Cell Ontogeny, and Epitope Structure. Immunity 48, 855-871 (2018).

2. Sok, D. \& Burton, D. R. Recent progress in broadly neutralizing antibodies to HIV. Nat. Immunol. 19, 1179-1188 (2018).

3. Stanfield, R. L., De Castro, C., Marzaioli, A. M., Wilson, I. A. \& Pantophlet, R. Crystal structure of the HIV neutralizing antibody $2 \mathrm{G} 12$ in complex with a bacterial oligosaccharide analog of mammalian oligomannose. Glycobiology 25, 412-419 (2015).

4. Clark, B. E. et al. A bacterial lipooligosaccharide that naturally mimics the epitope of the HIVneutralizing antibody $2 \mathrm{G} 12$ as a template for vaccine design. Chem. Biol. 19, 254-263 (2012).

5. Pantophlet, R. et al. Bacterially derived synthetic mimetics of mammalian oligomannose prime antibody responses that neutralize HIV infectivity. Nat. Commun. 8, 1601 (2017).

6. Trattnig, N., Blaukopf, M., Bruxelle, J.-F., Pantophlet, R. \& Kosma, P. Synthesis of an Undecasaccharide Featuring an Oligomannosidic Heptasaccharide and a Bacterial Kdo-lipid A Backbone for Eliciting Neutralizing Antibodies to Mammalian Oligomannose on the HIV-1 Envelope Spike. J. Am. Chem. Soc. 141, 7946-7954 (2019).

7. Kamboj, K. K., Kirchner, H. L., Kimmel, R., Greenspan, N. S. \& Schreiber, J. R. Significant variation in serotype-specific immunogenicity of the seven-valent Streptococcus pneumoniae capsular 
polysaccharide-CRM197 conjugate vaccine occurs despite vigorous T cell help induced by the carrier protein. J. Infect. Dis. 187, 1629-1638 (2003).

8. Pecetta, S. et al. Carrier priming effect of CRM197 is related to an enhanced B and T cell activation in meningococcal serogroup A conjugate vaccination. Immunological comparison between CRM197 and diphtheria toxoid. Vaccine 34, 2334-2341 (2016).

9. Kamboj, K. K., King, C. L., Greenspan, N. S., Kirchner, H. L. \& Schreiber, J. R. Immunization with Haemophilus influenzae type b-CRM(197) conjugate vaccine elicits a mixed Th1 and Th2 CD(4+) T cell cytokine response that correlates with the isotype of antipolysaccharide antibody. J. Infect. Dis. 184, 931-935 (2001).

10. Mawas, F., Feavers, I. M. \& Corbel, M. J. Serotype of Streptococcus pneumoniae capsular polysaccharide can modify the Th1/Th2 cytokine profile and IgG subclass response to pneumococal-CRM(197) conjugate vaccines in a murine model. Vaccine 19, 1159-1166 (2000).

11. Polonskaya, Z. et al. T cells control the generation of nanomolar-affinity anti-glycan antibodies. J. Clin. Invest. 127, 1491-1504 (2017).

12. Di Pasquale, A., Preiss, S., Tavares Da Silva, F. \& Garçon, N. Vaccine Adjuvants: from 1920 to 2015 and Beyond. Vaccines 3, 320-343 (2015).

13. Granoff, D. M., McHugh, Y. E., Raff, H. V., Mokatrin, A. S. \& Van Nest, G. A. MF59 adjuvant enhances antibody responses of infant baboons immunized with Haemophilus influenzae type $b$ and Neisseria meningitidis group C oligosaccharide-CRM197 conjugate vaccine. Infect. Immun. 65, 1710-1715 (1997).

14. Huang, Y.-L. et al. Carbohydrate-based vaccines with a glycolipid adjuvant for breast cancer. Proc. Natl. Acad. Sci. U. S. A. 110, 2517-2522 (2013).

15. Arias, M. A. et al. Glucopyranosyl Lipid Adjuvant (GLA), a Synthetic TLR4 Agonist, Promotes Potent Systemic and Mucosal Responses to Intranasal Immunization with HIVgp140. PLOS ONE 7, e41144 (2012).

16. Cauwelaert, N. D. et al. The TLR4 Agonist Vaccine Adjuvant, GLA-SE, Requires Canonical and Atypical Mechanisms of Action for TH1 Induction. PLOS ONE 11, (2016).

17. Coler, R. N. et al. A synthetic adjuvant to enhance and expand immune responses to influenza vaccines. PloS One 5, e13677 (2010).

18. Escolano, A., Dosenovic, P. \& Nussenzweig, M. C. Progress toward active or passive HIV-1 vaccination. J. Exp. Med. 214, 3-16 (2017).

19. Bruxelle, J.-F. et al. Serum alpha-mannosidase as an additional barrier to eliciting oligomannosespecific HIV-1-neutralizing antibodies. Sci. Rep. 10, 7582 (2020).

20. Alam, S. M. et al. Mimicry of an HIV broadly neutralizing antibody epitope with a synthetic glycopeptide. Sci. Transl. Med. 9, (2017).

21. Sanders, R. W. et al. A next-generation cleaved, soluble HIV-1 Env trimer, BG505 SOSIP.664 gp140, expresses multiple epitopes for broadly neutralizing but not non-neutralizing antibodies. PLOS Pathog. 9, e1003618 (2013). 
22. Kong, L. et al. Complete epitopes for vaccine design derived from a crystal structure of the broadly neutralizing antibodies PGT128 and 8ANC195 in complex with an HIV-1 Env trimer. Acta Crystallogr. D Biol. Crystallogr. 71, 2099-2108 (2015).

23. Pejchal, R. et al. A potent and broad neutralizing antibody recognizes and penetrates the HIV glycan shield. Science 334, 1097-1103 (2011).

24. Simonich, C. A. et al. HIV-1 Neutralizing Antibodies with Limited Hypermutation from an Infant. Cell 166, 77-87 (2016).

25. Freund, N. T. et al. Coexistence of potent HIV-1 broadly neutralizing antibodies and antibody-sensitive viruses in a viremic controller. Sci. Transl. Med. 9, (2017).

26. MacLeod, D. T. et al. Early Antibody Lineage Diversification and Independent Limb Maturation Lead to Broad HIV-1 Neutralization Targeting the Env High-Mannose Patch. Immunity 44, 1215-1226 (2016).

27. Sok, D. et al. A prominent site of antibody vulnerability on HIV envelope incorporates a motif associated with CCR5 binding and its camouflaging glycans. Immunity 45, 31-45 (2016).

28. Hu, J. K. et al. Murine Antibody Responses to Cleaved Soluble HIV-1 Envelope Trimers Are Highly Restricted in Specificity. J. Virol. 89, 10383-10398 (2015).

29. Kim, D. K. \& Hunter, P. Advisory Committee on Immunization Practices Recommended Immunization Schedule for Adults Aged 19 Years or Older - United States, 2019. MMWR Morb. Mortal. Wkly. Rep. 68, 115-118 (2019).

30. Robinson, C. L., Bernstein, H., Romero, J. R. \& Szilagyi, P. Advisory Committee on Immunization Practices Recommended Immunization Schedule for Children and Adolescents Aged 18 Years or Younger - United States, 2019. MMWR Morb. Mortal. Wkly. Rep. 68, 112-114 (2019).

31. Kurokawa, T. et al. Hemocyanin from the keyhole limpet Megathura crenulata (KLH) carries a novel type of N-glycans with Gal(beta1-6)Man-motifs. Eur. J. Biochem. 269, 5459-5473 (2002).

32. Barnes, C. O. et al. Structural characterization of a highly-potent V3-glycan broadly neutralizing antibody bound to natively-glycosylated HIV-1 envelope. Nat. Commun. 9, 1251 (2018).

33. Doores, K. J. et al. Two classes of broadly neutralizing antibodies within a single lineage directed to the high-mannose patch of HIV envelope. J. Virol. 89, 1105-1118 (2015).

34. Harmer, N. J. \& Chahwan, R. Isotype switching: Mouse IgG3 constant region drives increased affinity for polysaccharide antigens. Virulence 7, 623-626 (2016).

35. Damelang, T., Rogerson, S. J., Kent, S. J. \& Chung, A. W. Role of IgG3 in Infectious Diseases. Trends Immunol. 40, 197-211 (2019).

36. Perlmutter, R. M., Hansburg, D., Briles, D. E., Nicolotti, R. A. \& Davie, J. M. Subclass restriction of murine anti-carbohydrate antibodies. J. Immunol. Baltim. Md 1950 121, 566- 572 (1978).

37. Seabright, G. E., Doores, K. J., Burton, D. R. \& Crispin, M. Protein and Glycan Mimicry in HIV Vaccine Design. J. Mol. Biol. 431, 2223-2247 (2019). 
38. Trattnig, N. et al. Comparative Antigenicity of Thiourea and Adipic Amide Linked Neoglycoconjugates Containing Modified Oligomannose Epitopes for the Carbohydrate-Specific anti-HIV Antibody $2 \mathrm{G} 12$. Bioconjug. Chem. 30, 70-82 (2019).

39. Burnett, D. L., Reed, J. H., Christ, D. \& Goodnow, C. C. Clonal redemption and clonal anergy as mechanisms to balance B cell tolerance and immunity. Immunol. Rev. 292, 61-75 (2019).

40. Nguyen, D. N. et al. Oligomannose Glycopeptide Conjugates Elicit Antibodies Targeting the Glycan Core Rather than Its Extremities. ACS Cent. Sci. 5, 237-249 (2019).

41. Ringe, R. P. et al. Neutralizing Antibody Induction by HIV-1 Envelope Glycoprotein SOSIP Trimers on Iron Oxide Nanoparticles May Be Impaired by Mannose Binding Lectin. J. Virol. 94, (2020).

42. Liu, H. et al. Characterization and quantification of mouse mannan-binding lectins (MBL-A and MBLC) and study of acute phase responses. Scand. J. Immunol. 53, 489-497 (2001).

43. Schweighardt, B. et al. Development of an HIV-1 reference panel of subtype B envelope clones isolated from the plasma of recently infected individuals. J. Acquir. Immune Defic. Syndr. 1999 46, $1-11$ (2007).

44. Richman, D. D., Wrin, T., Little, S. J. \& Petropoulos, C. J. Rapid evolution of the neutralizing antibody response to HIV type 1 infection. Proc. Natl. Acad. Sci. U. S. A. 100, 4144-4149 (2003).

45. Sok, D. et al. Promiscuous glycan site recognition by antibodies to the high-mannose patch of gp120 broadens neutralization of HIV. Sci. Transl. Med. 6, 236ra63 (2014).

46. Hunt, L. A., Etchison, J. R. \& Summers, D. F. Oligosaccharide chains are trimmed during synthesis of the envelope glycoprotein of vesicular stomatitis virus. Proc. Natl. Acad. Sci. U. S. A. 75, 754-758 (1978).

\section{Declarations}

\section{ACKNOWLEDGMENTS}

We thank D. Carter and S. Maxwell (Infectious Disease Research Institute) for facilitating access to the GLA-SE adjuvant, M. Wabl and M. Wabl (Trianni Inc) for access to Trianni transgenic mice, B. Nguyen and G. Lin (Antibody Solutions) for coordinating the immunizations, and T. Wrin and Y. Lie (Monogram Biosciences) for organizing the neutralization assays reported here. We are grateful to J. Moore, A. Cupo and V. Cruz Portillo (Weill Cornell Medical College) for generously providing the BG505, B41, and C.ZA97 SOSIP trimers, to R. Sanders, R. Derking and T. Bijl (Amsterdam UMC) for the AMC008, ZM197M, and DU422 SOSIP trimers and to D. Blevins and J. Wulff (University of Victoria) for guidance with Biacore set up and assistance with data interpretation. We also thank K. Ng and S. Fu for technical support. Research reported in this publication was supported by the National Institute of Allergy and Infectious Diseases of the National Institutes of Health under award number R01Al134299 (to RP). A Scholar Award (award number 5268; to RP) from the Michael Smith Foundation for Health Research is also acknowledged. The funders had no role in study design, data collection and analysis, decision to publish, or preparation of the manuscript. 


\section{AUTHOR CONTRIBUTIONS}

J.-F.B., P.K., and R.P. conceived and designed the research. J.-F.B., T.K., N.T., Y.Y., and M.C. performed the research and analysed the data. J.-F.B., P.K., and R.P. supervised the research. J.-F.B. and R.P. wrote the manuscript. All authors commented on drafts of the manuscript and agreed to the final version.

\section{COMPETING FINANCIAL INTERESTS}

The authors declare no competing financial interests.

\section{Table}

Table 1. Binding avidity of the oligomannose-patch specific PGT128/130 antibodies correlates with glycoside loading density on NIT211.

\begin{tabular}{|c|c|c|c|}
\hline $\begin{array}{l}\text { mAb } \\
\text { (Fab fragment) }\end{array}$ & $\begin{array}{l}\mathrm{ka}\left(\mathrm{M}^{-1} \mathrm{~s}^{-1} \times 10^{3}\right) \\
(\mathrm{sd})\end{array}$ & $\begin{array}{l}\mathrm{kd}\left(\mathrm{s}^{-1} \times 10^{-3}\right) \\
(\mathrm{sd})\end{array}$ & $\begin{array}{l}\mathrm{K}_{\mathrm{D}}\left(\mathrm{M} \times 10^{-7}\right) \\
(\mathrm{sd})\end{array}$ \\
\hline PGT125 & $\begin{array}{l}5.64 \\
(2.44)\end{array}$ & $\begin{array}{l}1.99 \\
(2.05)\end{array}$ & $\begin{array}{l}3.09 \\
(2.03)\end{array}$ \\
\hline PGT126 & $\begin{array}{l}4.9 \\
(0.38)\end{array}$ & $\begin{array}{l}1.97 \\
(1.24)\end{array}$ & $\begin{array}{l}4.14 \\
(2.86)\end{array}$ \\
\hline PGT128 & $\begin{array}{l}4.53 \\
(2.71)\end{array}$ & $\begin{array}{l}3.22 \\
(2.33)\end{array}$ & $\begin{array}{l}6.78 \\
(1.09)\end{array}$ \\
\hline PGT130 & $\begin{array}{l}1.28 \\
(0.09)\end{array}$ & $\begin{array}{l}0.37 \\
(0.29)\end{array}$ & $\begin{array}{l}2.71 \\
(2.4)\end{array}$ \\
\hline
\end{tabular}

$\mathrm{mAb}$, monoclonal antibody; $\mathrm{ka}$, association constant; $\mathrm{kd}$, dissociation constant; $\mathrm{K}_{\mathrm{D}}$, equilibrium constant; sd, standard deviation.

\section{Figures}


A

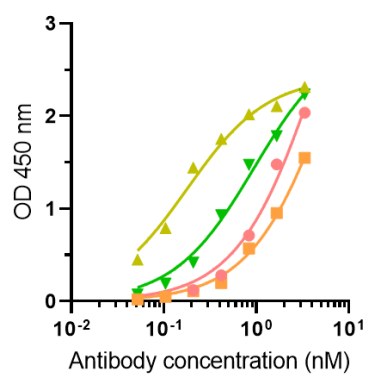

B

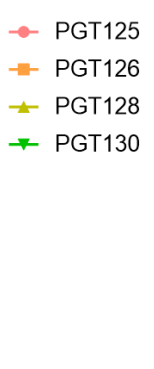

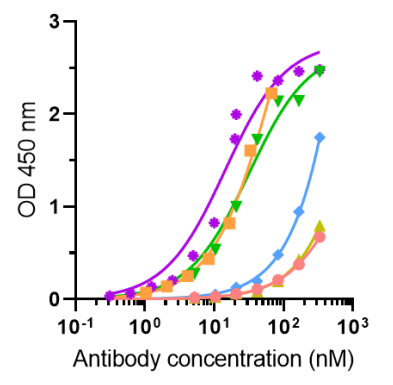

C

$\multimap$ BF520.1

- BG18

- PGDM12

* PGDM21

$\rightarrow$ PCDN-33A

* VRC41.01

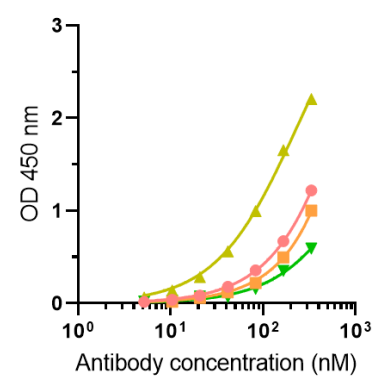

- BF520.1 gl

- $\mathrm{BG} 18 \mathrm{gl}$

- PCDN-33A gl

* PGT128/130 fam gl

\section{Figure 1}

NIT211 conjugate is recognized by oligomannose-specific bnAbs and their germline precursors. NIT211 (4.1 glycosides per CRM197) was coated as solid-phase antigen onto ELISA plate wells at $5 \mu \mathrm{g} / \mathrm{ml}$ in PBS and assayed for recognition by the different antibodies. All antibodies were expressed recombinantly as human IgG1. (A) Binding of PGT128/130 bnAb family members PGT125, PGT126, PGT128 and PGT130. (B) Binding of non-PGT128/130 family antibodies BF520.1, BG18, PCDN-33A, PGDM12, PGDM21, PCDN76-33A, VRC41.01. (C) Binding of inferred germline (gl) precursors BF520.1, BG18, PCDN-33A and the PGT128/130 family. Results are from single experiments performed with technical duplicates. 


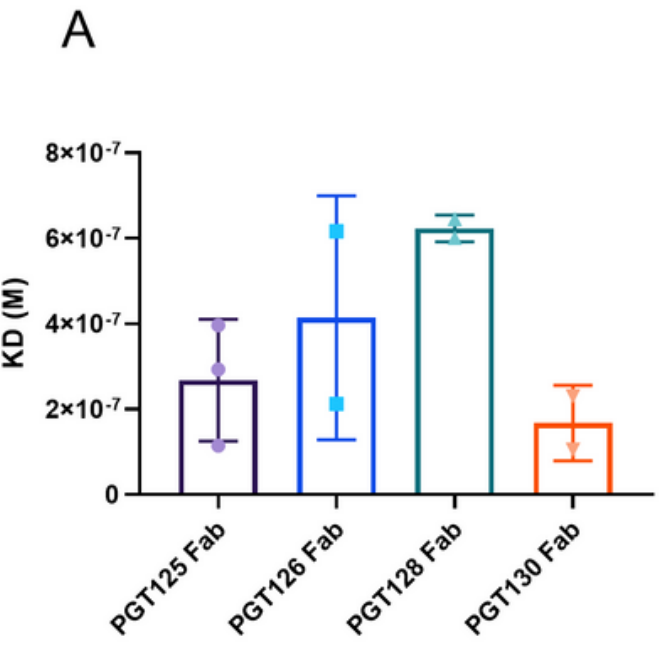

B

PGT125 Fab

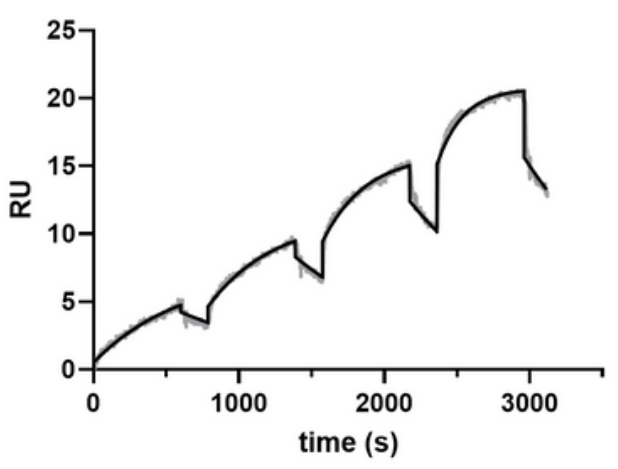

PGT128 Fab

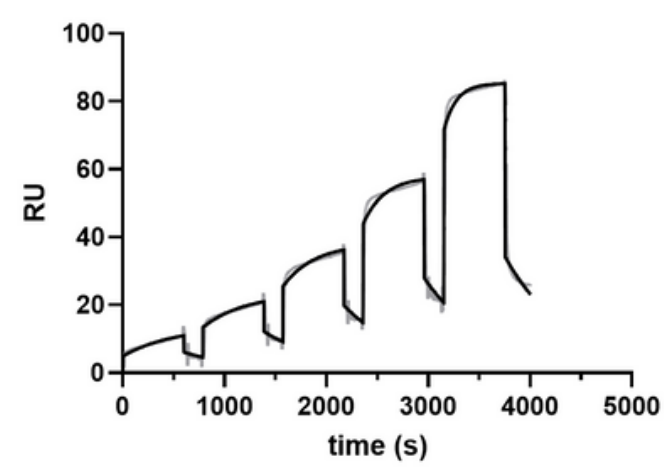

PGT126 Fab

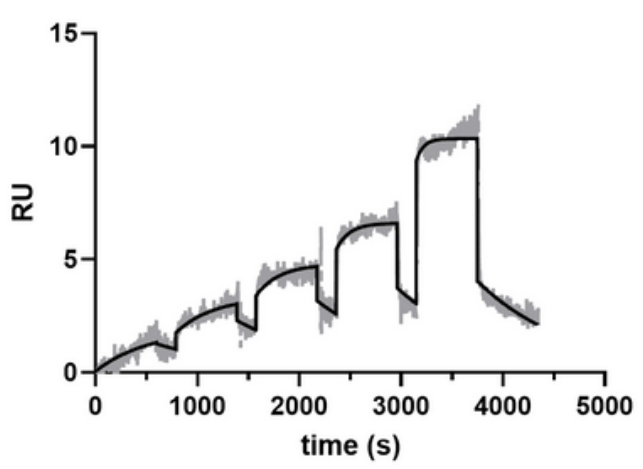

PGT130 Fab

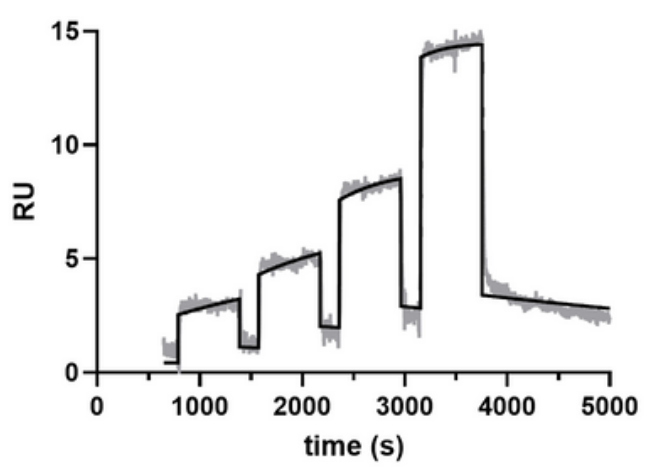

\section{Figure 2}

Binding affinities of PGT125, PGT126, PGT128 and PGT130 Fabs for NIT211 as measured by SPR. (A) Geometric mean of the association constant $(\mathrm{ka})$, dissociation constant $(\mathrm{kd})$ and equilibrium dissociation constant (KD) for the four PGT bnAbs. The results are from 2-3 independent experiments. (B) Representative sensorgrams of Fab binding to NIT211 using single-cycle kinetics. The calculcated 
association constant $(\mathrm{ka})$, dissociation constant $(\mathrm{kd})$ and $\mathrm{KD}$ are indicated. The black lines show the data (grey) fitted to a 1:1 binding model.

A PGT125 IgG

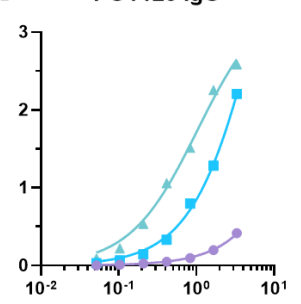

PGT128 IgG

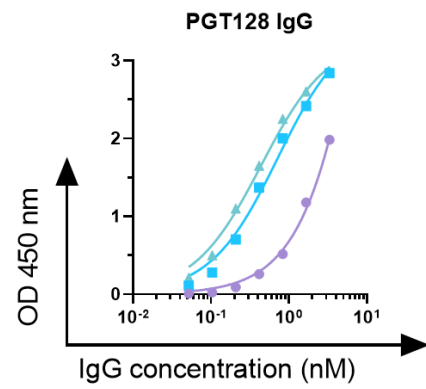

PGT126 IgG

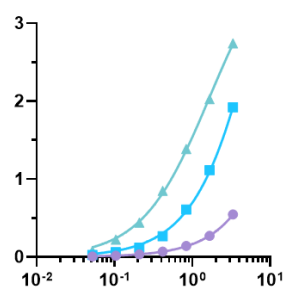

PGT130 IgG

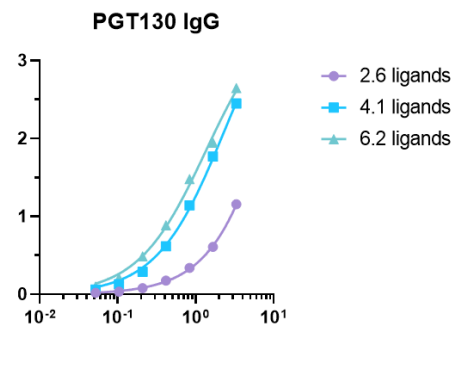

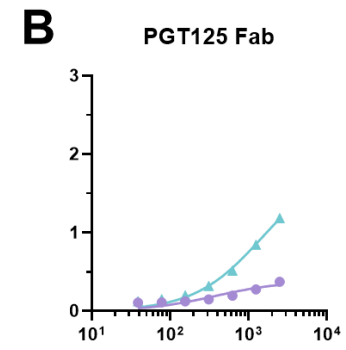

PGT128 Fab

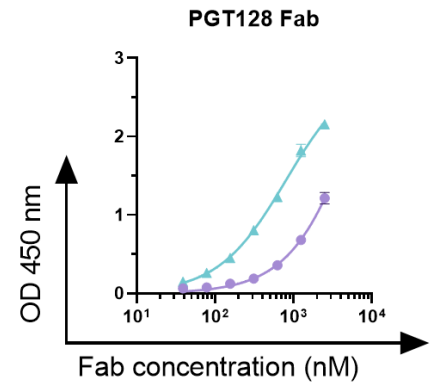

PGT126 Fab

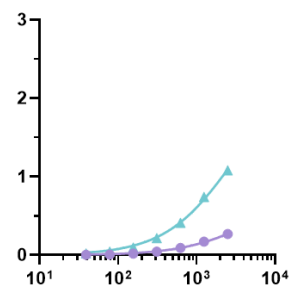

PGT130 Fab

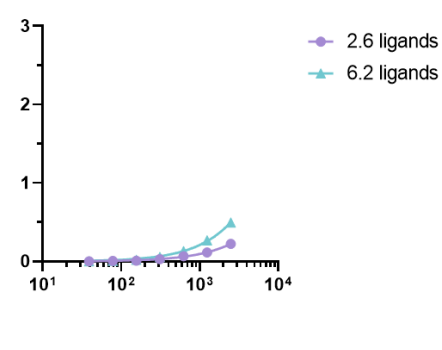

\section{Figure 3}

The relative binding affinities of PGT antibodies for NIT211 increase with increasing ligand density. Binding to NIT211 conjugates was assessed by ELISA. NIT211 (2.6 ligands, 4.1, and 6.2 ligands) was coated as solid-phase antigen onto ELISA plate wells at $5 \mu \mathrm{g} / \mathrm{ml}(82,79,76 \mathrm{nM}$ respectively) and assayed for antibody binding. (A) Binding of PGT125, PGT126, PGT128 and PGT130 IgG to NIT211 at three different densities of glycoside per CRM197. (B) Binding of PGT125, PGT126, PGT128 and PGT130 Fabs to NIT211 at two different densities of glycoside per CRM197.
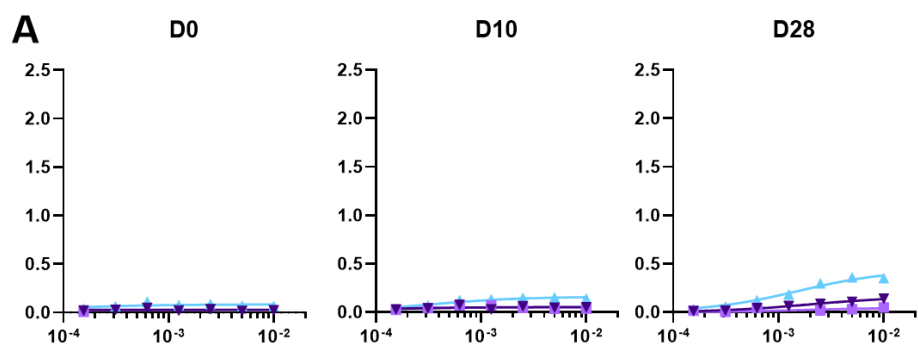

D49

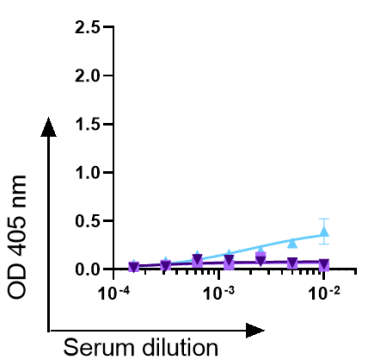

D119

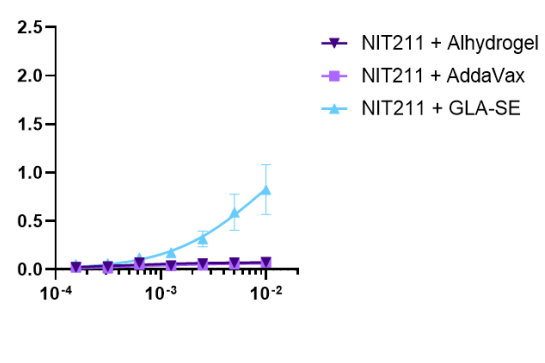

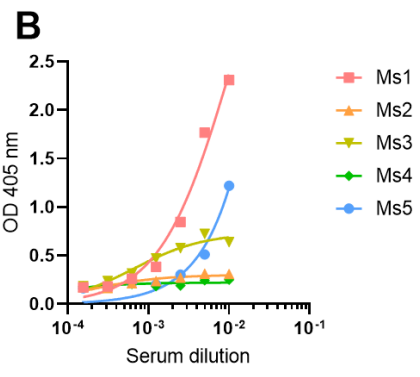

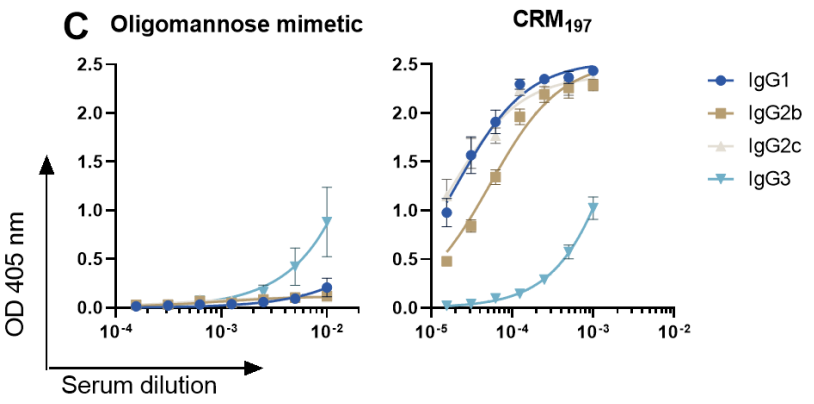

Figure 4 
Only animals administered GLA-SE-adjuvanted NIT211 mount an IgG response to the oligomannose mimetic that is of the lgG3 subclass. Trianni mice ( $n=5$ per group) were immunized subcutaneously (days $0,21,42$ and 105) and sera collected on day 0 prior to immunization and on days $10,28,49$, and 119 post-immunization. (A) Binding of total IgG antibodies in pre-immune and post-immune sera to BSAconjugated oligomannose mimetic. Binding curves represent mean values for the five animals in each immunization group, each tested in duplicate, with error bars denoting the standard deviation from the mean. (B) Individual IgG binding curves for NIT211 + GLA-SE immunized mice (Ms1 to 5). (C) IgG1, IgG2b, IgG2c and IgG3 antibody subclass responses in day 119 post-immune sera of NIT211 + GLA-SE immunized animals for the BSA-conjugated oligomannose mimetic in comparison to the CRM197 protein carrier.
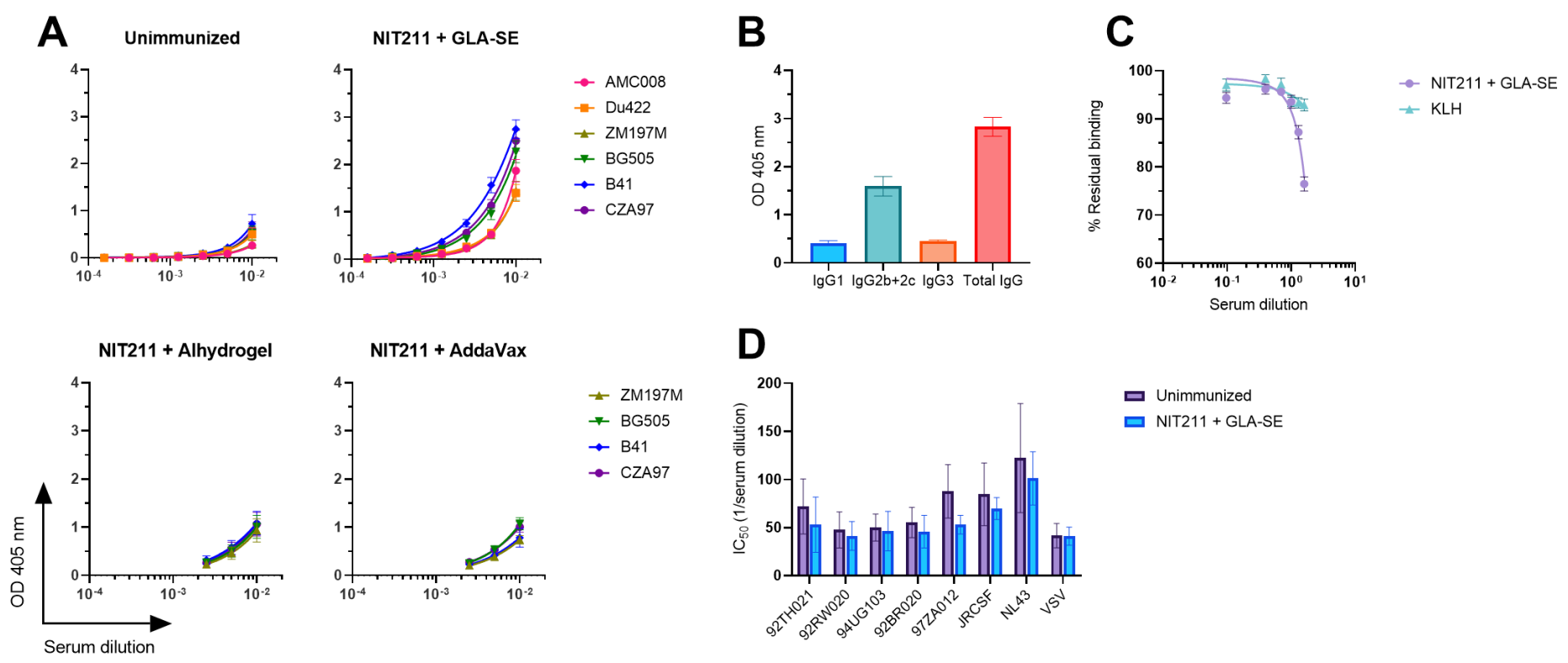

\section{Figure 5}

NIT211 formulated in GLA-SE elicits HIV-binding antibodies. (A) Binding of total IgG from pre-immune sera and day 119 post-immune sera from NIT211-immunized animal groups to SOSIP trimers AMC008 (subtype B), Du422 (subtype C), ZM197M (subtype C), BG505 (subtype A), B41 (subtype B), and CZA97 (subtype C). The HIS-tagged trimers were captured on nickel-coated ELISA plates at $5 \mu \mathrm{g} / \mathrm{ml}$ in PBS. (B) Level of IgG1, IgG2, IgG3 antibody subclass binding (1:100 dilution) to B41 SOSIP trimer in day 119 sera of NIT211 + GLA-SE immunized animals. (C) Residual binding of bnAb PGT128 to B41 SOSIP trimer following incubation with sera from NIT211 + GLA-SE immunized animals (day 119) vs sera from KLH + Alum/CpG ODN1826 immunized animals (day 34). (D) Day 119 sera from NIT211 + GLA-SE immunized animals and sera from a group of unimmunized animals were assessed for pseudovirus neutralization using a panel of seven diverse HIV-1 strains (92TH021, 92RW020, 94UG103, 92BR020, 97ZA012, JRCSF and NL4-3). Pseudotyped vesicular stomatitis virus (VSV) was used as a negative control. All graphs depict mean values for the serum samples from each group assayed. Error bars represent standard error from the mean. 


\section{Supplementary Files}

This is a list of supplementary files associated with this preprint. Click to download.

- BruxelleetalNatcomsupp.docx 